\title{
A FORMULATION OF MEMBRANE ELEMENTS WITH DRILLING DEGREES OF FREEDOM
}

\author{
By Masashi IURA*
}

\begin{abstract}
A new formulation of membrane elements with drilling degrees of freedom is presented. The Lagrange multiplier method is employed to obtain the modified functional. Since the independent rotational field is used, the present formulation is applicable to any shape of elements. The resulting stiffness matrix has negative diagonals associated with drilling rotations. This deficiency is remedied by introducing a positive parameter. It is shown that the numerical results are not affected by the value of the parameter. The present element has no zero-energy mode, and no locking phenomena are observed. Numerical results demonstrate an excellent performance of the membrane elements with drilling rotations

Keywords : drilling rotations, membrane element, FEM
\end{abstract}

\section{INTRODUCTION}

In-plane rotations about a normal to an element plane are referred to as drilling rotations. A key advantage of using a membrane element with drilling degrees of freedom (d.o.f.) is that a flat shell element consisting of the membrane and bending elements possesses six d. o. f. per node; the problem of singularity can be avoided without difficulty. When a membrane element without drilling d. of. is combined with a bending element to form a flat shell element, we have a zero stiffness in the direction of drilling rotations. In principle, there exist different approaches to avoid this singularity (see Zienkiewic $\mathrm{z}^{1)}$ ). In practice, however, these approaches lead to certain programming difficulties. Thus the presence of drilling d. o. f. is very attractive to engineers. The most commonly used approach to develop a membrane element with drilling d. o. f. is the addition of a fictitious set of rotation stiffness coefficients, as described by Zienkiewicz ${ }^{11}$. Although the fictitious stiffness is widely used, there remain problems to be improved. A number of work have been done to determine the real stiffness associated with the drilling rotations. In spite of much effort, there have been few elements which possess a correct rank, have no locking phenomena, and give satisfactory results.

Early attempts in the 1960's and 70's to develop membrane elements with drilling d. o.f. were unsuccessful. According to Allman"), the early formulations impose quite severe restrictions on the displacements in order to achieve compatibility; in particular, the drilling rotations of elements are constrained from changing to accommodate constant states of strain, so that convergence to an exact solution with mesh refinement is unassured. We refer to the references ${ }^{2)-1)}$ for a comprehensive review of these early papers. Allmanl has developed a triangular element with drilling d. o. f. on the basis of the

* Member of JSCE, Dr. Eng. . Associate Professor, Department of Civil and Structural Engineering. Tokyo Denki University (Hatoyama-machi, Hiki-gun, Saitama 350-03) 
principle of minimum potential energy. Although a level of success has been achieved in the work"), the element possesses the problem of zero-energy modes. Bergan and Felippa ${ }^{3)}$, based on the free formulation, have presented a triangular membrane element without having zero-energy modes. Since the above elements are restricted to the triangular ones, Cook $^{5)}$ has presented a quadrilateral element by introducing the coordinate transformation approach. After their papers have been published, a variety of membrane elements with drilling $\mathrm{d}$. o. f. have been proposed ${ }^{4)}{ }^{12)}$ to improve the performance of membrane elements. In these works, the displacement field is related to the rotational field. At first, the displacement field is expressed in terms of corner and midside nodal displacements. Then the midside nodal displacements are replaced by the corner nodal displacements and rotations. Finally, the corner nodal rotations are related to the corner drilling rotations.

Hughes and Brezzi ${ }^{13)}$ have presented variational principles employing independent rotational fields. Their formulation is attributable to the work of Naghdi ${ }^{14)}$ and Reissner ${ }^{15)}$ in which the symmetric condition for shear stresses are relaxed. The elements developed by Hughes and Brezzi are proved to give the converged solution. From numerical point of view, however, their elements are too stiff to give a satisfactory result. Herrmann ${ }^{16)}$, based on a couple-stress theory ${ }^{17)}$, has introduced a couple stress in addition to an antisymmetric shear stress to develop a membrane element with drilling d. o. f.. Although his formulation is straightforward, further research will be required to develop an efficient element which gives a satisfactory result. Suzuki and Kaneko ${ }^{18)}$, based on a micropolar theory, have also developed a membrane element possessing the in-plane rotation. The contribution of couple stresses are assumed to be small and neglected in the total strain energy. Since they have not analyzed any in-plane problem, the accuracy of their membrane element has not been confirmed. It will be shown in APPENDIX that the membrane elements, developed by Hughes and Brezzi ${ }^{13)}$ and Suzuki and Kaneko ${ }^{18)}$, are too stiff to give a satisfactory result.

In this paper, a new formulation of membrane elements with drilling d. o. f. is presented on the basis of classical elasticity, in which the symmetric condition of shear stresses is employed. The Lagrange multiplier method is used to obtain a new functional which leads to the definition of drilling rotations as the Euler-Lagrange equation. The rotational field employed is independent on the displacement field. Therefore, the present formulation is available for any shape of elements. The resulting stiffness matrix may have the negative diagonals associated with the drilling rotations. This deficiency is remedied by introducing a positive parameter. The numerical results are not affected by the value of the parameter. The present elements possess a correct rank and exhibit no locking problem under severe geometric distortion. Numerical examples demonstrate that the present element is usually more accurate than the existing ones. We consider herein a linear isotropic plane-stress problem. It is needless to say that the present formulation is applicable to a plane-strain problem with a slight modification.

\section{FORMULATION}

Let us consider a Cartesian system $(x, y)$. The drilling rotation about a normal to the element plane is defined by

$$
\theta=\frac{1}{2}\left(\frac{\partial v}{\partial x}-\frac{\partial u}{\partial y}\right)
$$

where $u$ and $v$ are displacement components in the direction of $x$ and $y$, respectively. The total potential energy function for a linear isotropic plane-stress problem may be written as ${ }^{19)}$

$$
\begin{aligned}
& U_{0}=\int\left[\frac{E h}{2\left(1-\nu^{2}\right)}\left\{\left(\frac{\partial u}{\partial x}\right)^{2}+\left(\frac{\partial v}{\partial y}\right)^{2}+2 \nu \frac{\partial u}{\partial x} \frac{\partial v}{\partial y}\right\}+\frac{1}{2} G h\left(\frac{\partial u}{\partial y}+\frac{\partial v}{\partial x}\right)^{2}\right] d A \\
& -\int[f u+g v] d A-\int_{s_{\sigma}}[\bar{f} u+\bar{g} v] d S
\end{aligned}
$$

where $E$ is Young's modulus, $G$ the shear modulus, $\nu$ Poisson's ratio, $h$ the thickness of material, and $f$ 
and $g$ are the body force components in the direction of $x$ and $y$, respectively; $\bar{f}$ and $\bar{g}$ denote the surface force components. Introducing the definition of drilling rotation, expressed by Eq. (1), into Eq. (2) with the use of the Lagrange multiplier method ${ }^{19)}$ and rearranging the resulting equation, we have

$$
\begin{aligned}
& U_{1}=\int\left[\frac{E h}{2\left(1-\nu^{2}\right)}\left\{\left(\frac{\partial u}{\partial x}\right)^{2}+\left(\frac{\partial v}{\partial y}\right)^{2}+2 \nu \frac{\partial u}{\partial x} \frac{\partial v}{\partial y}\right\}+\frac{1}{2} G h\left\{\frac{1}{2}\left(\frac{\partial u}{\partial y}+\frac{\partial v}{\partial x}\right)+\theta+\frac{\partial u}{\partial y}\right\}^{2}\right. \\
& \left.-M\left\{\theta-\frac{1}{2}\left(\frac{\partial v}{\partial x}-\frac{\partial u}{\partial y}\right)\right\}\right] d A-\int[f u+g v] d A-\int_{s_{\sigma}}[\bar{f} u+\bar{g} v] d S
\end{aligned}
$$

where $M$ is the Lagrange multiplier. The Euler-Lagrange equations of Eq. (3), derived from the condition such that $\delta U_{1}=0$, are written as

$$
\begin{aligned}
& \frac{E h}{\left(1-\nu^{2}\right)}\left(\frac{\partial^{2} u}{\partial x^{2}}+\nu \frac{\partial^{2} v}{\partial x \partial y}\right)+\frac{3}{4} G h\left(\frac{\partial^{2} u}{\partial y^{2}}+\frac{\partial^{2} v}{\partial x \partial y}\right)+\frac{3}{2} G h\left(\frac{\partial \theta}{\partial y}+\frac{\partial^{2} u}{\partial y^{2}}\right)-\frac{1}{2} \frac{\partial M}{\partial y}+f=0 \\
& \frac{E h}{\left(1-\nu^{2}\right)}\left(\frac{\partial^{2} v}{\partial y^{2}}+\nu \frac{\partial^{2} u}{\partial x \partial y}\right)+\frac{1}{4} G h\left(\frac{\partial^{2} v}{\partial x^{2}}+\frac{\partial^{2} u}{\partial x \partial y}\right)+\frac{1}{2} G h\left(\frac{\partial \theta}{\partial x}+\frac{\partial^{2} u}{\partial x \partial y}\right)+\frac{1}{2} \frac{\partial M}{\partial x}+g=0 \\
& \theta-\frac{1}{2}\left(\frac{\partial v}{\partial x}-\frac{\partial u}{\partial y}\right)=0 \\
& G h\left\{\frac{1}{2}\left(\frac{\partial u}{\partial y}+\frac{\partial v}{\partial x}\right)+\theta+\frac{\partial u}{\partial y}\right\}-M=0 \ldots \ldots \ldots \ldots \ldots \ldots \ldots \ldots \ldots \ldots \ldots \ldots \ldots \ldots \ldots \ldots \ldots \ldots \ldots \ldots \ldots \ldots \ldots \ldots \ldots \ldots \ldots \ldots \ldots \ldots \ldots \ldots \ldots \ldots
\end{aligned}
$$

Substituting Eq. (4·d) into Eq. (3) and eliminating the Lagrange multiplier $M$, we obtain the following functional :

$$
\begin{aligned}
U_{2}= & \int\left[\frac{1}{2\left(1-\nu^{2}\right)} E h\left\{\left(\frac{\partial u}{\partial x}\right)^{2}+\left(\frac{\partial v}{\partial y}\right)^{2}+2 \nu \frac{\partial u}{\partial x} \frac{\partial v}{\partial y}\right\}+\frac{1}{2} G h\left(\frac{\partial u}{\partial y}+\frac{\partial v}{\partial x}\right)^{2}\right. \\
& \left.-\frac{1}{2} \varkappa_{0} G h\left\{\theta-\frac{1}{2}\left(\frac{\partial v}{\partial x}-\frac{\partial u}{\partial y}\right)\right\}^{2}\right] d A-\int[f u+g v] d A-\int_{s_{\sigma}}[\bar{f} u+\bar{g} v] d S
\end{aligned}
$$

where $\varkappa_{0}=1$. When $\varkappa_{0}=-1$, the above functional takes the same form as that derived by Hughes and Brezzi $^{(3)}$, while the functional of Suzuki and Kaneko ${ }^{18)}$ is obtained by setting $\varkappa_{0}=-4$. The Euler-Lagrange equations of Eq. ( 5 ) are the linear momentum balance conditions and the definition of drilling rotation.

Since it follows from Eq. (1) that $\theta+\partial u / \partial y=\partial v / \partial x-\theta$, the underlined term in Eq. ( 3 ) can be replaced by $\partial v / \partial x-\theta$. The resulting functional gives the following Euler-Lagrange equations:

$$
\begin{aligned}
& \frac{E h}{\left(1-\nu^{2}\right)}\left(\frac{\partial^{2} u}{\partial x^{2}}+\nu \frac{\partial^{2} v}{\partial x \partial y}\right)+\frac{1}{4} G h\left(\frac{\partial^{2} u}{\partial y^{2}}+\frac{\partial^{2} v}{\partial x \partial y}\right)-\frac{1}{2} G h\left(\frac{\partial \theta}{\partial y}-\frac{\partial^{2} v}{\partial x \partial y}\right)-\frac{1}{2} \frac{\partial M^{*}}{\partial y}+f=0 \\
& \frac{E h}{\left(1-\nu^{2}\right)}\left(\frac{\partial^{2} v}{\partial y^{2}}+\nu \frac{\partial^{2} u}{\partial x \partial y}\right)+\frac{3}{4} G h\left(\frac{\partial^{2} v}{\partial x^{2}}+\frac{\partial^{2} u}{\partial x \partial y}\right)-\frac{3}{2} G h\left(\frac{\partial \theta}{\partial x}-\frac{\partial^{2} v}{\partial x^{2}}\right)+\frac{1}{2} \frac{\partial M^{*}}{\partial x}+g=0 \\
& \theta-\frac{1}{2}\left(\frac{\partial v}{\partial x}-\frac{\partial u}{\partial y}\right)=0 \\
& G h\left\{\frac{1}{2}\left(\frac{\partial u}{\partial y}+\frac{\partial v}{\partial x}\right)+\theta+\frac{\partial u}{\partial y}\right\}+M^{*}=0
\end{aligned}
$$

where $M^{*}$ is the Lagrange multiplier. The elimination of the Lagrange multiplier $M^{*}$, in a same manner described above, leads to the modified functional, the form of which is exactly the same as that expressed by Eq. ( 5 ). Therefore, in this paper, the functional $U_{2}$ is utilized to develop a membrane element with drilling rotations.

The functional $U_{2}$ might be regarded as the functional derived from the method of penalty functions (see Redd $\left.y^{20)}\right)$. However, there is a substantial difference between the present functional $U_{2}$ and the functional derived from the penalty function method. When we use the penalty function method, the penalty function or the constrain expressed by Eq. (1) should be introduced with a very large positive constant. And there is no unique way to determine the value of this empirical constant. As discussed in shell analysis by Kanok-Nukulchai ${ }^{21}$, the introduction of a penalty function makes the element stiffer. As a result, a selective integration has been often used to avoid an over-constrained situation. It will be shown later that 
there exist no over-constrained situation in the present element even if a full integration rule is employed to the constrain term.

Following the standard finite element method and using the functional $U_{2}$, we obtain the relationships between the generalized nodal displacements and nodal forces. The shape functions for displacements and rotations will be discussed in the next section. After assembling all the equations, we have an equation in a matrix form such as

$$
\left|\begin{array}{ll}
A_{11} & A_{12} \\
A_{21} & A_{22}
\end{array}\right|\left\{\begin{array}{c}
\boldsymbol{U} \\
\boldsymbol{\Theta}
\end{array}\right\}+\left|\begin{array}{ll}
B_{11} & B_{12} \\
B_{21} & B_{22}
\end{array}\right|\left\{\begin{array}{c}
\boldsymbol{U} \\
\boldsymbol{\theta}
\end{array}\right\}-\left|\begin{array}{ll}
C_{11} & C_{12} \\
C_{21} & C_{22}
\end{array}\right|\left\{\begin{array}{c}
\boldsymbol{U} \\
\boldsymbol{\theta}
\end{array}\right\}=\left\{\begin{array}{c}
\boldsymbol{F} \\
\mathbf{0}
\end{array}\right\}
$$

where $[A],[B]$ and $[C]$ are matrices associated with the first, second and third terms, respectively, of the first integral in Eq. ( 5 ); $\boldsymbol{U}$ and $\boldsymbol{\theta}$ denote the nodal displacements and rotations, respectively, and $\boldsymbol{F}$ denotes the nodal forces. Since the nodal rotations $\boldsymbol{\theta}$ do not appear in the first and second terms of the first integral in Eq. (5), we have $A_{12}=A_{21}=A_{22}=B_{12}=B_{21}=B_{22}=0$. Then Eq. ( 7 ) is rewritten as

$$
\left|\begin{array}{cc}
K_{11} & 0 \\
0 & 0
\end{array}\right|\left\{\begin{array}{l}
\boldsymbol{U} \\
\boldsymbol{\theta}
\end{array}\right\}-\left|\begin{array}{ll}
C_{11} & C_{12} \\
C_{21} & C_{22}
\end{array}\right|\left\{\begin{array}{c}
\boldsymbol{U} \\
\boldsymbol{\Theta}
\end{array}\right\}=\left\{\begin{array}{c}
\boldsymbol{F} \\
\mathbf{0}
\end{array}\right\}
$$

where $K_{11}=A_{11}+B_{11}$. Equation ( 8 ) shows that the total stiffness matrix may have the negative diagonals associated with nodal rotations. This deficiency may be remedied by modifying Eq. (8). In view of Eq.

( 8 ), we have

$$
\left|\begin{array}{ll}
C_{11} & C_{12} \\
C_{21} & C_{22}
\end{array}\right|\left\{\begin{array}{l}
\boldsymbol{U} \\
\boldsymbol{\theta}
\end{array}\right\}=\left\{\begin{array}{c}
\boldsymbol{G} \\
\mathbf{0}
\end{array}\right\}
$$

where

$$
\{\boldsymbol{G}\}=\left[K_{11}\right]\{\boldsymbol{U}\}-\{\boldsymbol{F}\}
$$

Using Eq. (9) and introducing the positive parameter $\alpha(>0)$, we can rewrite Eq. ( 8$)$ into the form

$$
\left|\begin{array}{cc}
K_{11} & 0 \\
0 & 0
\end{array}\right|\left\{\begin{array}{l}
\boldsymbol{U} \\
\boldsymbol{\theta}
\end{array}\right\}-(1+\alpha)\left|\begin{array}{ll}
C_{11} & C_{12} \\
C_{21} & C_{22}
\end{array}\right|\left\{\begin{array}{c}
\boldsymbol{U} \\
\boldsymbol{\theta}
\end{array}\right\}=\left\{\begin{array}{c}
\boldsymbol{F}-\alpha \boldsymbol{G} \\
\boldsymbol{0}
\end{array}\right\}
$$

The nodal rotations $\boldsymbol{\theta}$ are eliminated from Eq. (11) to yield the reduced form

$$
\left[K_{11}^{*}\right]\{\boldsymbol{U}\}=\{\boldsymbol{F}-\alpha \boldsymbol{G}\}
$$

where $K_{11}^{*}=K_{11}-(1+\alpha)\left(C_{11}-C_{12} C_{22}^{-1} C_{21}\right)$.

In order to develop the stiffness matrix with the degrees of freedom for drilling rotations, we rewrite Eq. (12) into the form

$$
\left|\begin{array}{cc}
K_{11}^{*} & \mathbf{0} \\
\mathbf{0} & \mathbf{0}
\end{array}\right|\left\{\begin{array}{c}
\boldsymbol{U} \\
\boldsymbol{\theta}
\end{array}\right\}=\left\{\begin{array}{c}
\boldsymbol{F}-\alpha \boldsymbol{G} \\
\mathbf{0}
\end{array}\right\}
$$

Substituting Eq. (9) into the right side of Eq. (13) and rearranging the resulting equation lead to

$$
\left|\begin{array}{cc}
K_{11}^{*}+\alpha C_{11} & \alpha C_{12} \\
\alpha C_{21} & \alpha C_{22}
\end{array}\right|\left\{\begin{array}{c}
\boldsymbol{U} \\
\boldsymbol{\theta}
\end{array}\right\}=\left\{\begin{array}{c}
\boldsymbol{F} \\
\mathbf{0}
\end{array}\right\}
$$

Then we have the total stiffness matrix which has the positive diagonals.

The parameter $\alpha$ should be chosen so that the eigenvalues of coefficient matrix, represented by Eq. (14), are positive except the zero-eigenvalues associated with the rigid displacements and rotations. In the present elements, there exist three zero-eigenvalues; two of them are associated with the rigid displacements and the rest is associated with the rigid rotation. The numerical experiments show that the parameter $\alpha$ can be taken in a wide range such that $10^{-5}<\alpha<10^{3}$. Note, however, that the solutions of Eq. (14), $\boldsymbol{U}$ and $\boldsymbol{\theta}$, are not affected by the value of $\alpha$. This is simply because Eq. (14) is equivalent to Eq.

\section{ISOPARAMETRIC ELEMENTS}

The present formulation is available for any shape of finite elements. In order to assess the performance of the element derived from the functional $U_{2}$, a 8-noded quadratic isoparametric element, as shown in 
Fig. 1, will be developed and tested. In the case of solving a shell problem in Sect. 4. (6) and a thick cantilever beam in APPENDIX, a 4-noded quadrilateral isoparametric element will be used. This is because an efficient 4 -noded bending element has been developed by many investigators. Since there is no difference for developing the elements between 4-noded and 8-noded elements, we will consider the 8-noded quadratic isoparametric element. We refer to the reference ${ }^{1)}$ for an isoparametric element.

The actual element geometry is mapped into a parent plane $(\xi, \eta)$ using the Serendipity shape functions, i. e.

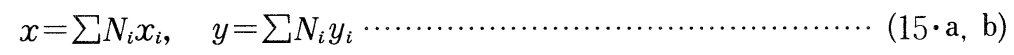

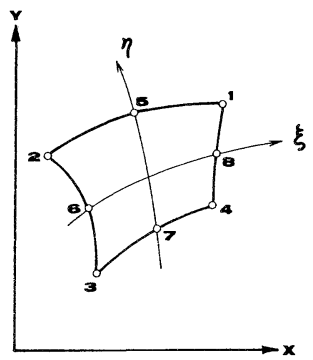

Fig. 1 8-Noded Isoparametric Element. where $i=1, \cdots, 8$ and

$$
\begin{aligned}
& N_{1}=\frac{1}{4} R S-\frac{1}{2} N_{5}-\frac{1}{2} N_{8}, \quad N_{2}=\frac{1}{4} \bar{R} S-\frac{1}{2} N_{5}-\frac{1}{2} N_{6}, \\
& N_{3}=\frac{1}{4} \bar{R} \bar{S}-\frac{1}{2} N_{6}-\frac{1}{2} N_{7}, \quad N_{4}=\frac{1}{4} R \bar{S}-\frac{1}{2} N_{7}-\frac{1}{2} N_{8}, \\
& N_{5}=\frac{1}{2} R^{*} S, \quad N_{6}=\frac{1}{2} \bar{R} S^{*}, \quad N_{7}=\frac{1}{2} R^{*} \bar{S}, \quad N_{8}=\frac{1}{2} R S^{*}, \\
& R=1+\xi, \quad S=1+\eta, \quad \bar{R}=1-\xi, \quad \bar{S}=1-\eta, \\
& R^{*}=1-\xi^{2}, \quad S^{*}=1-\eta^{2} .
\end{aligned}
$$

The element displacement and rotation components are defined in terms of element nodal displacements and rotations, $u_{i}, v_{i}$ and $\theta_{i}$, as

$$
u=\sum N_{i} u_{i}, \quad v=\sum N_{i} v_{i}, \quad \theta=\sum N_{i} \theta_{i}
$$

where $i=1, \cdots, 8$. Substituting Eqs. (15) and (17) into Eq. (5) and following the standard finite element procedure, we obtain the stiffness matrix for this problem. The Gaussian quadrature rule is applied to Eq.

(5). It should be noted that no reduced integration is used for the 8-noded element and that the selective reduced integration is used for the 4-noded element. As a result, the stiffness matrix has a correct rank for both the 4-noded and 8-noded elements. It can be shown later that the present elements do not exhibit locking phenomena; such problems have been often encountered in the case of using a full integration.

\section{NUMERICAL EXAMPLES}

A variety of test problems have been analyzed to evaluate the performance of the proposed membrane element. The purpose of these problems is to examine the numerical performance of the element with respect to (1) shear locking, (2) sensitivity to element distortion, (3) accuracy in stress calculations and (4) the in-plane rotations. As an application to shell problems, a pinched cylinder with end diaphragms has been solved.

The present finite elements have compatible displacements and rotations, and can recover all constant strain states. A convergence to exact solutions, therefore, is always assured by using consistent mesh refinement. The stresses in each element are obtained from the present displacement field. If more than one element meets at a node point of the finite element mesh, then the average value of the element stresses is quoted.

The value of $\alpha$ is taken as 0.1 in this paper except for a shell problem. Note, once again, that the numerical results are not affected by the value of $\alpha$. The same numerical results are obtained even though the value of $\alpha$ is equal to zero.

\section{(1) Uniform tension}

This example as shown in Fig. 2 is presented to show that the present element can recover the constant stress states and that no drilling rotations occur under the uniform tension. This test is one of the so called patch test ${ }^{1)}$. The material properties are $E=1 \mathrm{kgf} / \mathrm{cm}^{2}, \nu=0.3$, and $h=1 \mathrm{~cm}$. Table 1 shows the 


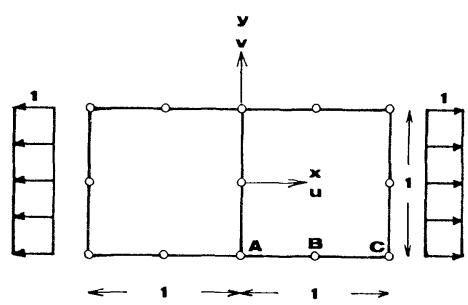

Fig. 2 Uniform Tension (unit : cm, kgf).
Table 1 Numerical Results of Uniform Tension (unit: $\mathrm{cm}, \mathrm{kgf}, \mathrm{rad}$ ).

\begin{tabular}{|c|c|c|c|c|}
\hline Point & U & v & $\sigma_{x}$ & $\theta$ \\
\hline A & $\begin{array}{l}0.0 \\
(0.0)\end{array}$ & $\begin{array}{l}0.1500 \times 10^{0} \\
(0.1500)\end{array}$ & $\begin{array}{l}0.1000 \times 10^{+1} \\
(1.000)\end{array}$ & $\begin{array}{l}0.0 \\
(0.0)\end{array}$ \\
\hline B & $\begin{array}{l}0.5000 \times 10^{0} \\
(0.5000)\end{array}$ & $\begin{array}{l}0.1500 \times 10^{0} \\
(0.1500)\end{array}$ & $\begin{array}{l}0.1000 \times 10^{+1} \\
(1.000)\end{array}$ & $\begin{array}{l}0.3538 \times 10^{-8} \\
(0.0000)\end{array}$ \\
\hline C & $\begin{array}{l}0.1000 \times 10^{+1} \\
(1.000)\end{array}$ & $\begin{array}{l}0.1500 \times 10^{0} \\
(0.1500)\end{array}$ & $\begin{array}{l}0.1000 \times 10^{+1} \\
(1.000)\end{array}$ & $\begin{array}{l}0.8935 \times 10^{-8} \\
(0.0000)\end{array}$ \\
\hline
\end{tabular}

Note: () denotes the exact value.

numerical results. A good agreement between numerical results and exact ones has been obtained. Due to rounding errors of digital computers, the drilling rotations do not become zero. These small values for drilling rotations, however, can be regarded as zero.

\section{(2) Thick cantilever beam under a tip load}

This example as shown in Fig. 3 has been used extensively in the finite element literature. The cantilever is subjected to a parabolically distributed force and has the material properties : $E=30000 \mathrm{kgf} / \mathrm{cm}^{2}, \nu=$ 0.25 , and $h=1 \mathrm{~cm}$. The numerical results for both regular and irregular meshes are listed in Table 2 , where the results using the other elements are also shown. An analytical answer of $0.35583 \mathrm{~cm}$ is used to
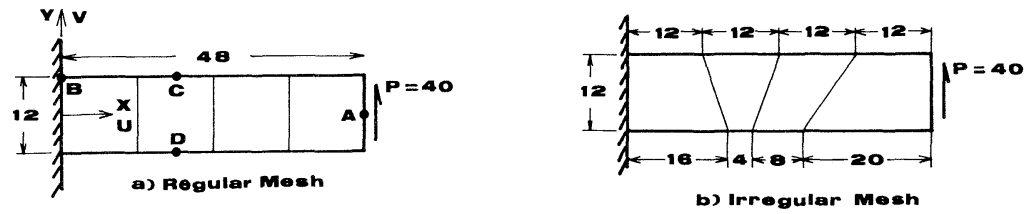

Fig. 3 Thick Cantilever Beam (unit: cm, kgf).

Table 2 Normalized Results of Thick Cantilever Beam.

\begin{tabular}{|c|c|c|c|c|c|c|c|c|c|}
\hline \multirow[b]{2}{*}{ Element } & \multirow{2}{*}{$\begin{array}{l}\text { No. of } \\
\text { d.o.f. }\end{array}$} & \multicolumn{2}{|c|}{$\mathrm{V}_{\mathrm{A}}$} & \multicolumn{2}{|c|}{$\sigma_{\mathrm{xB}}$} & \multicolumn{2}{|c|}{$\sigma_{\mathrm{xC}}$} & \multicolumn{2}{|c|}{$\sigma_{\mathrm{xD}}$} \\
\hline & & Reg. & Irreg. & Reg. & Irreg. & Reg. & Irreg. & Reg. & Irreg. \\
\hline Al1man [2] & 81 & 0.9164 & $-\cdots$ & $\ldots$ & ----- & $\ldots$ & $\ldots$ & $\ldots \ldots$ & $-\cdots$ \\
\hline Bergan [3] & 81 & 0.9772 & ----- & ---- & ------ & ---- & $-\cdots-\cdots$ & ----- & ----- \\
\hline \multirow{2}{*}{ MacNeal [4] } & $30^{\mathrm{a}}$ & 0.9776 & 0.9761 & -- - & ----- & -- - & $-\cdots$ & $---\cdots$ & -.-- \\
\hline & $30^{\mathrm{b}}$ & 0.9581 & 0.8867 & -.-- & $\ldots$ & $\ldots$ & $\ldots$ & ----- & 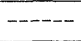 \\
\hline $\begin{array}{l}\text { Cook }[5] \\
(\mathrm{AQ} \text { Elem。) }\end{array}$ & 30 & 0.9226 & 0.9496 & 0.8750 & 0.8813 & 1.0000 & 1.0720 & 1.0000 & 0.9200 \\
\hline Sabir [6] & 81 & 0.9707 & - & $-\cdots$ & ------ & $-\cdots--$ & $-\cdots-$ & ----- & - - - \\
\hline Bergan [7] & 81 & 0.9995 & $\ldots$ & $\cdots$ & $\ldots$ & ---- & 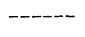 & $\ldots-$ & $\ldots-\cdots$ \\
\hline $\begin{array}{l}\text { Cook }[8] \\
(\lambda=0.2)\end{array}$ & 30 & 1.0490 & 0.9167 & 1.0010 & 0.9338 & 1.1580 & 0.9080 & 1.1460 & 1.5320 \\
\hline Lee [9] & 81 & 0.8313 & ----- & $-\cdots$ & $--\cdots$ & $-\cdots--$ & ---- & $-\cdots--$ & $-\cdots--$ \\
\hline Al1man [11] & 81 & 0.9089 & ---- & -...- & ------ & - - - - & - - - - & ----- & -...-- \\
\hline \multirow{2}{*}{ Yunus [12] } & $30^{c}$ & 1.0140 & 0.9861 & 0.9888 & 0.9238 & 1.1040 & 1.0180 & 1.0760 & 1.4640 \\
\hline & $30^{\mathrm{d}}$ & 0.9308 & 0.9673 & 0.8988 & 0.8788 & 1.0000 & 1.0120 & 1.0000 & 1.0160 \\
\hline 01 son [23] & 81 & 0.8383 & $-\cdots-$ & ---- & $-\cdots$ & ----- & $\ldots-\cdots$ & $\ldots$ & -- \\
\hline \multirow{2}{*}{ Present } & 39 & 0.9578 & ----- & 0.9475 & ----- & 0.9955 & - - - & 0.9955 & $-\cdots-$ \\
\hline & 69 & 0.9827 & 0.9784 & 0.9933 & 1.0100 & 1.0000 & 1.0090 & 1.0000 & 0.9372 \\
\hline
\end{tabular}

Reg. : Regular Elements Irreg. : Irregular Elements

a: Spurious modes are not controled.

b: Spurious modes are controled.

c: Mixed AT.

d: Mixed AQ. 
normalize the tip displacement. The stresses $\sigma_{X B}, \sigma_{X C}$ and $\sigma_{X D}$ are normalized by the exact solutions of $-80.0 \mathrm{kgf} / \mathrm{cm}^{2},-50.0 \mathrm{kgf} / \mathrm{cm}^{2}$, and $50.0 \mathrm{kgf} / \mathrm{cm}^{2}$, respectively. Blanks in Table 2 denote that these values are not available from the existing literature.

As discussed by Timoshenko and Goodier ${ }^{22}$, there is no unique way to idealize a fixed boundary condition. The drilling rotations at fixed ends have been often constrained in order to suppress the zero-energy modes. As pointed out by Bergan and Felippa ${ }^{3)}$, the continuum mechanics definition expressed by Eq. (1) is non-zero at the fixed ends due to the term $\partial v / \partial x$. In this paper, the fixed boundary conditions are idealized by constraining the displacement components, $u$ and $v$, at the fixed ends.

The finite element developed by Bergan and Nygard ${ }^{7}$, using 81 d. o. f., shows a good performance in this example. In their formulation, however, there remain a problem to decide the value of parameter which affects the numerical results. The finite element, called "Mixed AT" element developed by Yunus, Saigal and $\mathrm{Cook}^{12)}$, gives an excellent performance except the stress $\sigma_{X D}$. The "Mixed AT" element, however, tends to lock in a thin beam problem under severe geometric distortion, as shown in Sect. 4. (3). The performance of the present element is quite remarkable for both regular and irregular meshes. Since the use of only two elements (No. of d. o.f. $=39$ ) can not treat with the bending problem of irregular mesh, we can not fill in blanks associated with the present element in Table 2.

The exact drilling rotation at the tip is $0.1075 \times 10^{-1} \mathrm{rad}$, while the present element gives the values of $0.1076 \times 10^{-1} \mathrm{rad}$ and $0.1081 \times 10^{-1} \mathrm{rad}$ for the drilling rotation of regular and irregular meshes, respectively. Note that the error is $0.56 \%$ even for the irregular mesh.

\section{(3) Thin cantilever beam under pure bending}

This example has been used to evaluate the element capabilities for presenting the pure bending under severe geometric distortion. The cantilever beam is subjected to the end forces, as shown in Fig. 4 , and has the material properties: $E=3 \times 10^{7} \mathrm{kgf} / \mathrm{cm}^{2}, \quad \nu=0.3$, and $h=1 \mathrm{~cm}$. The numerical results, normalized against the analytical solution of $1.8 \times 10^{-4} \mathrm{~cm}$, are presented in Table 3 along with results from other elements. It is observed that most of the existing elements, including the "Mixed AT" element developed by Yunus, Saigal and $\mathrm{Cook}^{12)}$, tend to lock in this problem. The advantage of the present formulation is seen from this problem. The beam theory gives the value of $0.6000 \times 10^{-4}$ rad for drilling rotation at the tip. The value of $0.5532 \times 10^{-4} \mathrm{rad}$ for drilling rotation was obtained by the present element.

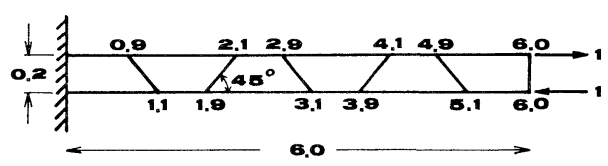

Fig. 4 Thin Cantilever Beam (unit: cm, kgf).

Table 3 Normalized Results of Thin Cantilever Beam.

\begin{tabular}{|l|l|}
\hline Element & Tip Deflection \\
\hline $\begin{array}{l}\text { Yunus [12] } \\
\text { Mixed AT })\end{array}$ & 0.50 \\
\hline $\begin{array}{c}\text { Yunus [12] } \\
\text { (Mixed AQ) }\end{array}$ & 0.85 \\
\hline Allman * & 0.21 \\
\hline $\begin{array}{l}\text { Pian and * } \\
\text { Sumihara }\end{array}$ & 0.16 \\
\hline Cook & 0.82 \\
\hline Present & 0.92 \\
\hline
\end{tabular}

* These values are given in [12]

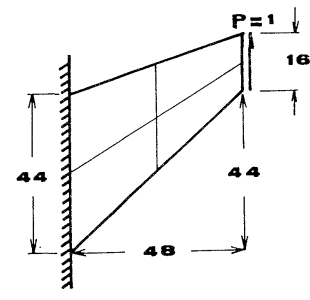

Fig. 5 Cook's Problem (unit: cm, kgf).

Table 4 Normalized Results of Cook's Problem.

\begin{tabular}{|l|l|l|}
\hline Element & $\begin{array}{c}\text { No。of } \\
\text { d。o.f。 }\end{array}$ & Tip Deflection \\
\hline Bergan [3] & 75 & 0.9377 \\
\hline Bergan [7] & 75 & 0.9665 \\
\hline Cook [8] & 75 & 0.9682 \\
\hline \multirow{2}{*}{ Yunus [1.2] } & $75^{a}$ & 0.9594 \\
\cline { 2 - 4 } & $75^{b}$ & 0.9728 \\
\hline Present & 63 & 0.9506 \\
\hline
\end{tabular}

a : Mixed AT, b : Mixed AQ 


\section{(4) Cook's problem}

This problem is a good exercise of element's ability to model membrane situations with skewed meshes. A skewed plate is clamped on one end and subjected to a uniformly distributed in-plane bending load on the other end, as shown in Fig. 5. Since there exist no analytical solutions, a finite element converged solution of $23.91 \mathrm{~cm}$ is used to normalize the results, which are shown in Table 4. The material properties are $E=$ $1 \mathrm{kgf} / \mathrm{cm}^{2}, \quad \nu=1 / 3$, and $h=1 \mathrm{~cm}$. No distinct differences among the existing results are found in this problem.

\section{(5) Pure bending of a square plate}

This example was proposed by Allman ${ }^{2}$. The square plate is subjected to linearly varying distributed forces, as shown in Fig. 6. Since the analytical solutions are available for this case, the numerical results for drilling rotations have been compared with exact solutions. The exact solutions for this problem are expressed as $^{2)}$

$$
u=2\left(\frac{q_{0}}{E}\right) \frac{x y}{L}, \quad v=-\left(\frac{q_{0}}{E}\right) \frac{x^{2}+\nu y^{2}}{L}, \quad \theta=-2\left(\frac{q_{0}}{E}\right) \frac{x}{L} .
$$

Allman ${ }^{2 l}$ has introduced the additional constrains for symmetric conditions in order to suppress the zero-energy modes. Since the present element has no zero-energy modes, the exact boundary and symmetric conditions are imposed in this paper.

Table 5 shows the numerical results for displacements and drilling rotations. It is observed that a complete agreement between numerical and exact solutions has been obtained for both displacements and rotations, when we utilize four 8 -noded elements developed herein.

\section{(6) Shell problem}

The main advantage of the use of elements with drilling rotations is prominent in an analysis of shell structures. Since an efficient 4-noded bending element with a correct rank has been developed without having locking phenomena, we analyze a shell problem using a 4-noded element. The example as shown in Fig. 7 is a pinched cylinder. This is a challenging problem in a finite element shell analysis and has been solved by many investigators. The bending element is developed on the basis of the work of Bathe and

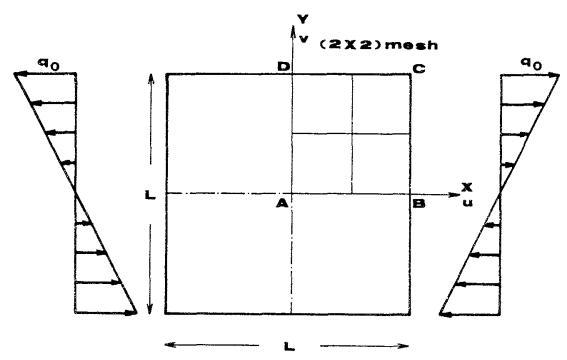

Table 5 Normalized Results of Square Plate.

\begin{tabular}{|l|l|l|l|l|l|}
\hline Element & $\begin{array}{c}\text { No. of } \\
d_{0} \text { o.f. }\end{array}$ & $\frac{E u_{c}}{\sigma_{0} L}$ & $\frac{E v_{c}}{\sigma_{0} L}$ & $\frac{E \theta_{c}}{\sigma_{0}}$ & $\frac{\sigma_{x c}}{\sigma_{0}}$ \\
\hline Allman [2] & 75 & 0.494 & -0.320 & -0.982 & 0.996 \\
\hline Allman [11] & 75 & 0.482 & -0.311 & -0.813 & 0.941 \\
\hline Present & 63 & 0.500 & -0.325 & -1.000 & 1.000 \\
\hline Exact & & 0.500 & -0.325 & -1.000 & 1.000 \\
\hline
\end{tabular}

Fig. 6 Square Plate.

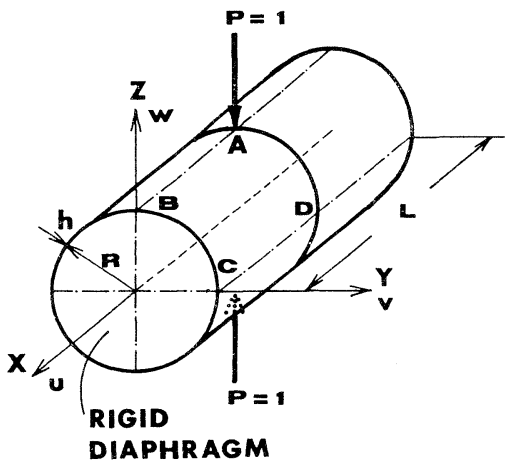

Fig. 7 Pinched Cylinder (unit: kgf).

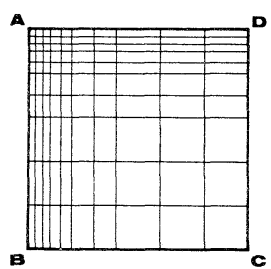

(A)

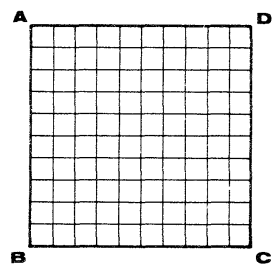

(B)

Fig. 8 Mesh Patterns. 
Table 6 Normalized Results of Pinched Cylinder.

\begin{tabular}{|l|c|l|}
\hline & Mesh A & Mesh B \\
\hline Bathe [24] & - & 0.830 \\
Liu [26] & - & 0.854 \\
Simo [27] & - & 0.823 \\
Present & 0.965 & 0.830 \\
\hline
\end{tabular}

Table 7 Normalized Results of Thick Cantilever Beam.

\begin{tabular}{|l|c|c|}
\hline & 4 Elements & 16 Elements \\
\hline Hughes and Brezzi [13] & 0.863 & 0.953 \\
Suzuki and Kaneko [18] & 0.740 & 0.900 \\
Present & 0.994 & 0.997 \\
\hline
\end{tabular}

Dvorkin $^{24)}$. The 4-noded membrane element is developed on the basis of Eq. ( 5 ). The value of $\alpha$ is taken as zero and the stiffness matrix is not reduced in this example. The length of the cylinder is $L=600 \mathrm{~cm}$, the thickness of shell is $h=3 \mathrm{~cm}$, and the radius is $R=300 \mathrm{~cm}$. The material properties are $E=3 \times$ $10^{6} \mathrm{kgf} / \mathrm{cm}^{2}, \nu=0.3$, and the two mesh patterns are used, as shown in Fig. 8. The numerical results, normalized against the exact solution of $0.1825 \times 10^{-4} \mathrm{~cm}$, are presented in Table 6 . Solutions obtained using another 4-noded element are also shown. It is seen from Table 6 that numerical results are sensitive to the mesh patterns. It is natural for a finite element analysis to use fine meshes around a concentrated force. The use of mesh (A), therefore, yields the accurate result. The best result in Mesh (B) has been obtained by Liu, Law, Lam and Belytschko ${ }^{26)}$ in which the stabilization matrices have been employed. When compared with their formulation, the present formulation seems to be simple and straightfoward. Note that the present element yields the better result than the mixed element developed by Simo, Fox and Rifai $^{27)}$ and that no fictitious stiffness has been used in this formulation to assemble the overall elements which possess six degrees of freedom per node.

\section{CONCLUDING REMARKS}

A new formulation for membrane elements that possess the drilling d. o. f. has been presented in this paper. We have assumed, on the basis of classical elasticity, that the shear stresses are symmetric and no external couples exist. As is well known ${ }^{17)}$, if a body couple, denoted by $t$, is applied, the shear stresses are no longer symmetric. When there exist the body couple, $t$, the surface couple, $\bar{t}$, and the couple stresses, $\mu_{x}$ and $\mu_{y}$, we obtain the following equilibrium equation and the mechanical boundary condition associated with the drilling rotation :

$$
\frac{\partial \mu_{x}}{\partial x}+\frac{\partial \mu_{y}}{\partial y}+\tau_{x y}-\tau_{y x}+t=0, \quad \mu_{x} n_{x}+\mu_{y} n_{y}=\bar{t}
$$

where $n_{x}$ and $n_{y}$ denote the direction cosines of the unit normal drawn outwards on the boundary. It is observed from Eq. $(19 \cdot \mathrm{b})$ that the assigned surface couple, $\bar{t}$, should be equal to zero if there exist no couple stresses. Lee and $\mathrm{Yoo}^{9)}$, and Yoshida, Amemiya and Masuda ${ }^{25.5}$ have analyzed the example in which a concentrated couple is applied. Since their elements have been developed on the basis of the classical elasticity, the mechanical boundary condition associated with the drilling rotation would be violated.

It is clear that the present formulation is not available for the problem in which a concentrated couple is applied. Such a problem might be analyzed by using a couple-stress theory or employing symmetric and skewsymmetric shear stresses. However, most of the problems encountered can be analyzed by using the classical elasticity. Numerical results for a variety of examples demonstrate that the present elements show superior results even under severe geometric distortion, and a better performance compared to other existing elements.

\section{ACKNOWLEDGEMENT}

This study is supported in part by the Grant-in Aid for Scientific Research from the Japanese Ministry of Education, Science and Culture.

\section{APPENDIX}

In this appendix, the thick cantilever beam, as illustrated in Fig. 3(a), is analyzed again for showing the 
numerical effect of the parameter $x_{0}$ introduced in Eq. (5). As described in Section 2, Hughes and Brezzi $^{13 .}$ have derived the functional with $x_{0}=-1$, while the membrane element developed by Suzuki and Kaneko: is obtained by setting $\varkappa_{0}=-4$. The normalized tip deflections obtained using the same data in Section 4. are listed in Table 7. It is shown, as described by Hughes and Brezzi ${ }^{13)}$, that both of the elments developed by Hughes and Brezzi ${ }^{13)}$ and Suzuki and Kaneko ${ }^{18)}$ give poor results.

\section{REFERENCES}

1) Zienkiewicz, O. C. : The Finite Element Method, 3rd ed., McGraw-Hill, 1977.

2) Allman, D. J. : A compatible triangular element including vertex rotations for plane elasticity analysis, Computers and Structures, 19, No.1-2, 1-8, 1984.

3) Bergan, P. G. and Felippa, C. A. : A triangular membrane element with rotational degrees of freedom, Comput. Meths. Appl. Mech. Engrg., 50, 25-69, 1985.

4) MacNeal, R. H. and Harder, R. L. : A refined four-noded membrane element with rotational degrees of freedom, Computers and Structures, 28, No. 1, 75-84, 1988

5) Cook, R. D. : On the Allman triangle and a related quadrilateral element, Computers and Structures, 22, No. 6, $1065-1067$. 1986.

6) Sabir. A. B. : A rectangular and a triangular plane elasticity element with drilling degree of freedom, Proc. of 2nd Int. Conf. on Variational Methods in Eng., Ed. by C. A. Brebbia, 9-17 9-26. Univ. of Southampton, Springer-Verlag, 1985.

7) Bergan, P. G. and Nygard, M. K. : A quadrilateral membrane element with rotational freedoms, Proc. of ICCM, Ed. by G. Yagawa and S. N. Atluri, I-41 I-50. Tokyo, Springer-Verlag, 1986.

8) Cook, R. D. : A plane hybrid element with rotational D. O.F. and adjustable stiffness. Int. J. Numer. Methods Eng., 24, 1499-1508. 1987.

9) Lee, S. C. and Yoo, C. H. : A novel shell element including in-plane torque effect, Computers and Structures, 28, No.4. 505-522, 1988.

10) Yunus, S. M. : A study of different hybrid elements with and without rotational D. O.F. for plane stress/plane strain problems, Computers and Structures, 30, No.5, 1127-1133, 1988.

11) Allman, D. J. : Evaluation of the constant strain triangle with drilling rotations, Int. J. Numer. Methods Eng. , 26, 2645-2655. 1988.

12) Yunus, S. M., Saigal, S. and Cook, R. D. : On improved hybrid finite elements with rotational degrees of freedom, Int. J. Numer. Methods Eng., 28, 785-800. 1989.

13) Hughes, T. J. R. and Brezzi, F. : On drilling degrees of freedom, Comput. Meths. Appl. Mech. Engrg., 72, 105-121, 1989.

14) Naghdi, P. M. : On a variational theorem in elasticity and its application to shell theory, J. Appl. Mech., 647-653. 1964.

15) Reissner, E. : A note on variational principles in elasticity, Int. J. Solids and Structures, 1. 93-95, 1965.

16) Herrmann, L. R. : Mixed finite elements for couple-stress analysis, Hybrid and Mixed F. E. M., Ed. by S. N. Atluri, R. H. Gallagher and O. C. Zienkiewicz, John Wiley and Sons, 1983.

17) Malvern, L. E. : Introduction to the Mechanics of a Continuous Medium, Prentice-Hall, Inc., 1969.

18) Suzuki, T. and Kaneko, H. : A large deflection analysis for buckling and postbuckling behavior of plate elements of structural members by the finite element method, Transactions of Arch. Inst. of Japan, No. 316, 9-17, 1982.

19) Washizu, K. : Variational Methods in Elasticity and Plasticity, 3 rd edn., Pergamon Press, 1982.

20) Reddy, J. N. : Applied Functional Analysis and Variational Methods in Engineering. McGraw-Hill, 1986.

21) Kanok-Nukulchai, W. : A simple and efficient finite element for general shell analysis, Int. J. Numer. Methods Eng., 14. 179-200. 1979.

22) Timoshenko, S. and Goodier, J. N. : Theory of Elasticity, 3rd ed., McGraw-Hill, 1970.

23) Olson, M. D. and Bearden, T. W. : A simple flat triangular shell element revisited, Int. J. Numer. Methods Eng., 14, 51-68, 1979.

24) Bathe, K. J. and Dvorkin, E. N. : A formulation of general shell elements--The use of mixed interpolation of tensorial components, Int. J. Numer. Methods Eng., 22, 697-722, 1986.

25) Yoshida, Y., Amemiya, E. and Masuda, N. : A flat finite element for thin shell analysis derived by assumed stress approach, Proc. of JSCE, No.211, 19-28, 1973.

26) Liu, W. K., Law, E. S., Lam, D. and Belytschko, T. : Resultant-stress degenerated-shell element, Comput. Meths. Appl. Mech. Engrg. , 55, 259-300, 1986.

27) Simo, J.C. Fox, D. D. and Rifai, M. S. : On a stress resultant geometrically exact shell model. Part 2 : The linear theory; Computational aspects, Comput. Meths. Appl. Mech. Engrg., 73, 53-92, 1989.

(Received May 31 1989) 